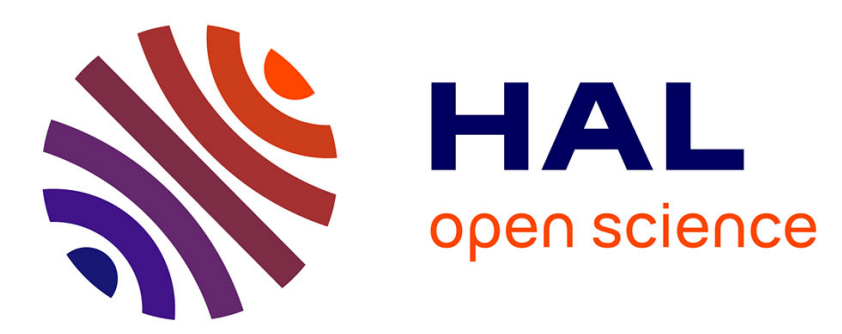

\title{
Characterization at the Molecular Level using Robust Biochemical Approaches of a New Kinase Protein
}

\author{
Béatrice Vallée, Michel Doudeau, Fabienne Godin, Hélène Bénédetti
}

\section{To cite this version:}

Béatrice Vallée, Michel Doudeau, Fabienne Godin, Hélène Bénédetti. Characterization at the Molecular Level using Robust Biochemical Approaches of a New Kinase Protein. Journal of visualized experiments: JoVE, 2019, 148, pp.e59820. 10.3791/59820 . hal-02282630

\section{HAL Id: hal-02282630 \\ https://hal.science/hal-02282630}

Submitted on 17 Jun 2020

HAL is a multi-disciplinary open access archive for the deposit and dissemination of scientific research documents, whether they are published or not. The documents may come from teaching and research institutions in France or abroad, or from public or private research centers.
L'archive ouverte pluridisciplinaire HAL, est destinée au dépôt et à la diffusion de documents scientifiques de niveau recherche, publiés ou non, émanant des établissements d'enseignement et de recherche français ou étrangers, des laboratoires publics ou privés. 


\section{Characterization at the Molecular Level using Robust Biochemical Approaches of a New Kinase Protein}

AUTHORS

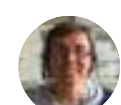

Béatrice Vallée $^{1}$

M

Michel Doudeau ${ }^{1}$

$\mathrm{F}$

AFFILIATIONS

${ }^{1}$ Centre de Biophysique Moléculaire, CNRS, UPR 4301, University of Orléans and INSERM

CORRESPONDING AUTHOR

Béatrice Vallée

beatrice.vallee@cnrs.fr

\section{Abstract}

Extensive whole genome sequencing has identified many Open Reading Frames (ORFs) providing many potential proteins. These proteins may have important roles for the cell and may unravel new cellular processes. Among proteins, kinases are major actors as they belong to cell signaling pathways and have the ability to switch on or off many processes crucial to the fate of the cell, such as cell growth, division, differentiation, motility, and death.

In this study, we focused on a new potential kinase protein, LIMK2-1. We demonstrated its existence by Western Blot using a specific antibody. We evaluated its interaction with an upstream regulating protein using coimmunoprecipitation experiments. Coimmunoprecipitation is a very powerful technique able to detect the interaction between two target proteins. It may also be used to detect new partners of a bait protein. The bait protein may be purified either via a tag

engineered to its sequence or via an antibody specifically targeting it. These protein complexes may then be separated by SDS-PAGE (Sodium Dodecyl Sulfate PolyAcrylamide Gel) and identified using mass spectrometry. Immunoprecipitated LIMK2-1 was also used to test its kinase activity in vitro by $\gamma\left[{ }^{32} \mathrm{P}\right]$ ATP labeling. This well-established assay may use many different substrates, and mutated versions of the bait may be used to assess the role of specific residues. The effects of pharmacological agents may also be evaluated since this technique is both highly sensitive and quantitative. Nonetheless, radioactivity handling requires particular caution. Kinase activity may also be assessed with specific antibodies targeting the phospho group of the modified amino acid. These kinds of antibodies are not commercially available for all the phospho modified residues. 


\section{Introduction}

For many decades, numerous signaling pathways have been elucidated and their involvement in crucial cellular processes such as cell division, differentiation, motility, programmed cell death, immunity and neurobiology, has been shown. Kinases play a significant role in these signaling pathways as they often finely regulate their activation or inactivation and are part of transient versatile complexes that respond to external stimuli $^{1,2,3}$. Mutation and dysregulation of kinases often lead to diseases in humans, and they have therefore become one of the most important drug targets over the past forty years ${ }^{4}$.

In this context, it is important to be able to detect kinase interaction with their upstream regulators or downstream substrates and to identify new partners. Affinity purification and immunoprecipitation are very powerful techniques for the isolation of protein complexes 5 . The bait protein or kinase may be tagged with a specific peptide sequence allowing the use of commercial beads covalently coupled with antibodies targeting the peptide. This material permits a high reproducibility in experiments $^{6,7,8}$. Endogenous proteins may also be immunoprecipitated using antibodies targeting directly the bait protein. The antibodies may be cross-linked to Protein A or Protein $\mathrm{G}$ agarose beads or simply incubated with these beads prior to adding lysate. Lysis buffers must be optimized to allow protein solubilization without losing interaction and to avoid protein degradation. A major drawback of this approach is that the interaction is detected upon cell lysis; therefore, transient or weak interactions, together with those requiring subcellular context may be missed. Other techniques may be used to work directly in the cell such as Proximity Ligation Assay (PLA) ${ }^{9}$, in vivo cross-linking-assisted affinity purification (XAP) ${ }^{10}$, Bioluminescence Resonance Energy Transfer (BRET) or Förster Resonance Energy Transfer (FRET) ${ }^{11,12}$. Furthermore, immunoprecipitation is not appropriate to determine the thermodynamic constants of the binding, for which physical techniques such as Surface Plasmon Resonance, Isothermal Titration Calorimetry or Microscale Thermophoresis are required ${ }^{13,14}$.

Kinase activity may be assessed using multiple techniques. Herein, we focused on phospho-specific antibodies and in vitro $\gamma\left[{ }^{32} \mathrm{P}\right]$ ATP (Adenosine TriPhosphate) labeling. Phospho-specific antibodies target the phosphate modification of a particular residue within a protein. They may be used in Western Blot or ELISA (Enzyme-Linked ImmunoSorbent Assay) after cell lysis, for immunohistochemistry, and also on intact cells using flow cytometry or immunofluorescence. Their drawbacks may include their lack of specificity, which can be evaluated using a mutated version of the target protein, and their not being commercially available for all proteins. In vitro $\gamma\left[{ }^{32} \mathrm{P}\right]$ ATP labeling is a very robust, well-established and highly sensitive method $^{15}$. Immunoprecipitated or recombinant proteins may be used, and different substrates may be tested. The effects of drugs may also be assessed as this method is quantitative. Its major drawback is that the radioactivity associated with the approach requires handling with caution. Alternative methods are also possible based on the measurement of fluorescent or luminescent peptide substrates and taking advantage of altered fluorescent/luminescent properties upon phosphorylation. Such methods also allow high throughput, which is required, for example, in the screening of molecules that may be potential inhibitors of the target kinase. Indeed, kinases represent one of the largest classes of drug targets pursued by pharmaceutical companies $^{16}$.

In this study, we focused on the LIMK2-1 protein (LIMK2-1 stands for Lin11, Isle1, Mec3 Kinase isoform 2-1). The LIMK2 kinase protein was first described in $1995^{17}$. Three isoforms of LIMK2 are produced by alternative splicing: LIMK2a, LIMK2b and LIMK2-1. At present, LIMK2-1 has only been described at 
the mRNA level in a single study ${ }^{18}$. Herein, we characterize this potential new kinase protein at the molecular level using robust biochemical approaches. Firstly, we demonstrate that LIMK2-1 is indeed synthesized. Similar to its two counterparts, LIMK2a and LIMK2b, it interacts with the upstream kinase ROCK (Rho-associated protein kinase). We show LIMK2-1 has a kinase activity on Myelin Basic Protein (MBP), but not on cofilin, the canonical substrate of LIM kinases.

\section{Protocol}

\section{Cell preparation for transfection}

CAUTION: All the steps of the cell culture must be performed in a dedicated laboratory, and cells are manipulated within a Class 2 microbiological cabinet.

1. Seed HEK-293 (Human Embryonic Kidney) cells in $\varnothing 10$ $\mathrm{cm}$ plates in $10 \mathrm{~mL}$ of DMEM (Dulbecco's Modified Eagles Medium) supplemented with $10 \%$ fetal calf serum. Culture for 3 to 5 days under $5 \% \mathrm{CO}_{2}$, at $37^{\circ} \mathrm{C}$, until the cells reach $\sim 90 \%$ confluence.

2. Treat $\varnothing 10 \mathrm{~cm}$ plates with Collagen $\mathrm{R}$ to increase cell adhesion to the plastic plates.

1. Add $5 \mathrm{~mL}$ of a solution of Collagen $\mathrm{R}, 200$-fold diluted in Phosphate Saline Buffer (PBS) in a $\varnothing 10 \mathrm{~cm}$ plate. Overlay the liquid over the whole surface of the plate.

2. Incubate at room temperature for at least $1 \mathrm{~h}$ within the biosafety cabinet.

3. Remove collagen solution, and discard it. Add $5 \mathrm{~mL}$ of PBS, spread it all over the surface of the plate, remove it and discard it. Repeat this wash once.
4. Add $8 \mathrm{~mL}$ of DMEM supplemented with $10 \%$ fetal calf serum. Keep the prepared plates within the biosafety cabinet.

3. Take HEK-293 plates from step 1.1 within the biosafety cabinet. Remove the medium from the plates and discard it in a dedicated bleach trash. Add $2 \mathrm{~mL}$ of supplemented DMEM, and flush the cells to detach them using the flow of a $1 \mathrm{~mL}$ micropipette, taking care to avoid making foam.

4. Collect the cells in a $15 \mathrm{~mL}$ tube and add $4 \mathrm{~mL}$ of supplemented DMEM. Homogenize with a $10 \mathrm{~mL}$ pipette, by pipetting up and down 3 times.

5. Take $2 \mathrm{~mL}$ of this cell solution and add them to collagen-treated plates containing supplemented DMEM from step 1.2.4.

6. Grow for 24 hours in the incubator at $5 \% \mathrm{CO}_{2}$ and $37^{\circ} \mathrm{C}$. Cells should be $50-80 \%$ confluent at the time of transfection.

\section{Transient transfections}

1. Remove the medium from the plates, discard it in a dedicated bleach trash, and add $10 \mathrm{~mL}$ of fresh supplemented DMEM. Put back the plates into the incubator at $37^{\circ} \mathrm{C}$ while preparing the transfection mixture.

2. In a $15 \mathrm{~mL}$ tube, add $450 \mu \mathrm{L}$ of a $10 \mathrm{mM}$ Tris- $\mathrm{HCl} \mathrm{pH}$ 7.5/1 mM EDTA (Tris stands for Tris (hydroxymethyl)aminomethane, and EDTA for ethylenedinitrilotetraacetic acid) solution and $50 \mu \mathrm{L}$ of a $2.5 \mathrm{M} \mathrm{CaCl}_{2}$ solution. Mix by inversion.

3. Add $10 \mu \mathrm{g}$ of plasmidic DNA (Deoxyribonucleic acid) prepared from a midi preparation on a liquid culture of 
bacteria transformed with the dedicated plasmid. Mix by inversion.

4. Under smooth agitation on a vortex, add $500 \mu \mathrm{L}$ of BES buffered saline $2 \mathrm{x}$ concentrate (composition: BES, 10.7 g/L, NaCl, $16.0 \mathrm{~g} / \mathrm{L}, \mathrm{Na}_{2} \mathrm{HPO}_{4}, 0.27 \mathrm{~g} / \mathrm{L} ; \mathrm{BES}$ stands for $\mathrm{N}, \mathrm{N}$-Bis(2-hydroxyethyl)-2-aminoethanesulfonic acid, $\mathrm{N}, \mathrm{N}-\mathrm{Bis}$ (2-hydroxyethyl)taurine) slowly drop by drop.

5. Incubate for at least $15 \mathrm{~min}$ (up to $45 \mathrm{~min}$ ) at room temperature within the biosafety cabinet. Do not vortex, do not mix! Move the tubes very carefully not to disturb the complex formation between DNA and calcium phosphate.

6. Take the plates from step 2.1 to the safety cabinet. Add the DNA complexes very carefully, drop by drop, onto the cells all over the surface of the plate.

7. Incubate for 24 to 72 hours in the incubator at $37^{\circ} \mathrm{C}$. Usually maximum protein expression is reached within 48 hours.

\section{Lysis}

NOTE Work on ice, and with cold buffers to prevent protein degradation.

1. Prepare lysis buffer: $50 \mathrm{mM}$ Tris- $\mathrm{HCl}, \mathrm{pH} 7.5,100 \mathrm{mM}$ NaCl, 5 mM EDTA, 0.1\% Triton X-100, 50 mM NaF, 10 $\mathrm{mM}$ sodium pyrophosphate, $1 \mathrm{mM} \mathrm{Na}_{3} \mathrm{VO}_{4}, 20 \mathrm{mM}$ p-nitrophenyl phosphate, $20 \mathrm{mM} \beta$-glycerophosphate, $10 \mu \mathrm{g} / \mathrm{mL}$ aprotinin, $0.05 \mu \mathrm{g} / \mathrm{mL}$ okaidic acid, $1 \mu \mathrm{g} / \mathrm{mL}$ leupeptin, and $1 \mathrm{mM}$ PMSF (phenylmethylsulfonyl fluoride). Around $4 \mathrm{~mL}$ of lysis buffer are required for each transfected plate.

2. Remove plates with transfected cells from the incubator. Put them on ice.
NOTE From this step, it is possible to work on a "normal" bench.

3. Remove the media, and discard it in a dedicated bleach trash.

4. Wash twice with $3 \mathrm{~mL}$ of cold PBS: add $3 \mathrm{~mL}$ of PBS on the side of the plate drop by drop to avoid detaching transfected cells, spread all over the surface of the plate, remove PBS and discard it; repeat this step once more. At the end, remove carefully the rest of PBS by tilting the plate to prevent lysis buffer dilution for next step.

5. Add $500 \mu \mathrm{L}$ of cold lysis buffer on the transfected washed cells. Spread it all over the surface of the plate.

6. Incubate for $10 \mathrm{~min}$ on ice. From time to time (at least twice), spread again the buffer all over the surface of the plate.

7. Scrap the cells and collect them in a microcentrifuge tube.

8. Centrifuge for $10 \mathrm{~min}$ at $10,000 \mathrm{xg}$ at $4{ }^{\circ} \mathrm{C}$.

9. Collect supernatant in a new microcentrifuge tube. This fraction corresponds to the lysate (whole cell extract). Discard the pellet which corresponds to cell membrane debris.

10. Collect an aliquot of this fraction to a new microcentrifuge tube (around $50 \mu \mathrm{L}$ ). This fraction corresponds to the "TOTAL fraction" or "Cell Lysate" or "Whole Cell extract" allowing analysis of whether transfected proteins are expressed by Western Blot.

NOTE At this point, samples can be directly used for Western Blot analyses. Laemmli buffer must be added to the sample, which is then heated at $95 \mathrm{C}$ for $5 \mathrm{~min}$, centrifuged at $10,000 \times \mathrm{g}$ for $5 \mathrm{~min}$, and loaded on appropriate 
SDS-PAGE. Samples may also be stored at $-80^{\circ} \mathrm{C}$.

\section{Immunoprecipitation}

1. Gently resuspend agarose beads coupled with the appropriate antibody: HA (Human influenza hemagglutinin), Flag, or GFP (Green Fluorescent Protein) by smooth inversion.

2. Cut the end of a $200 \mu \mathrm{L}$ tip from a micropipette to allow beads to enter the tip. Pipette the beads up and down several times to saturate the tip to ensure to take the correct volume of beads.

3. Take $40 \mu \mathrm{L}$ of beads in a microcentrifuge tube.

4. Add $500 \mu \mathrm{L}$ of TENET ( $30 \mathrm{mM}$ Tris- $\mathrm{HCl} \mathrm{pH} \mathrm{7.5,} 120 \mathrm{mM}$ NaCl, 5 mM EDTA, 1\% Triton X-100) buffer. Mix by inversion. Centrifuge for $2 \mathrm{~min}$ at $1,000 \mathrm{x} \mathrm{g}$ at $4{ }^{\circ} \mathrm{C}$. Remove carefully supernatant and discard it. Add $500 \mu$ $\mathrm{L}$ of TENET. Incubate for at least 1 hour at $4{ }^{\circ} \mathrm{C}$ on a rotating wheel.

NOTE This pre-incubation step in TENET allows to reduce non-specific interactions and so to decrease background signal.

5. Wash the beads twice with $500 \mu \mathrm{L}$ of lysis buffer.

1. Centrifuge $2 \mathrm{~min}$ at $1,000 \mathrm{xg}$ at $4^{\circ} \mathrm{C}$.

2. Remove carefully supernatant buffer, and discard it.

NOTE Take care not to aspirate beads during these washing steps.

3. Add $500 \mu \mathrm{L}$ of lysis buffer. Homogenize by inverting the tube.

4. Repeat steps 4.5.1- 4.5.3.
6. Centrifuge for $2 \mathrm{~min}$ at $1,000 \mathrm{x} \mathrm{g}$ at $4^{\circ} \mathrm{C}$. Carefully remove supernatant buffer, and discard it.

7. Incubate beads with the lysate from step 3.9 for 2 to 4 hours at $4^{\circ} \mathrm{C}$ on a rotating wheel.

8. Wash immunoprecipitated beads.

NOTE At this point, the immunoprecipitated beads may be washed five times with the lysis buffer, and then eluted with Laemmli buffer. The eluate may be used for Western Blot analyses or stored at -80 C. Alternatively, beads may be washed twice with the lysis buffer and then three times with the kinase buffer to perform $\gamma\left[{ }^{32} \mathrm{P}\right]$ ATP labeling.

\section{Coimmunoprecipitation analyses}

1. Wash immunoprecipitated beads from step 4.7 with the lysis buffer.

1. Centrifuge for $2 \mathrm{~min}$ at $1,000 \mathrm{xg}$ in a refrigerated centrifuge at $4{ }^{\circ} \mathrm{C}$.

2. Carefully remove the supernatant, and discard it.

3. Add $500 \mu \mathrm{L}$ of lysis buffer. Homogenize by inverting the tube.

4. Repeat steps 5.1.1-5.1.3 four times.

2. Elution

1. Centrifuge for $2 \mathrm{~min}$ at $1,000 \mathrm{x} \mathrm{g}$ in a refrigerated centrifuge at $4^{\circ} \mathrm{C}$.

2. Carefully remove the supernatant, and discard it. Remove the last drops of supernatant with a Hamilton syringe in order to avoid aspiration of the beads, and discard the supernatant.

3. Add $40 \mu \mathrm{L}$ of $4 \mathrm{x}$ Laemmli buffer ( $200 \mathrm{mM}$ Tris/HCl pH 6.8, 4\% SDS, 40\% glycerol, $0.5 \mathrm{M}$ 
$\beta$-mercaptoethanol, 0.02\% bromophenol blue).

Homogenize by gently tapping the tube.

4. Incubate for $5 \mathrm{~min}$ at room temperature.

5. Centrifuge for $5 \mathrm{~min}$ at $10,000 \mathrm{xg}$ at room temperature.

6. With a Hamilton syringe, remove the supernatant and collect it in a new microcentrifuge tube. This fraction corresponds to the "Eluate", which may be stored at $-80^{\circ} \mathrm{C}$, or directly analyzed by Western Blot.

\section{Kinase assay}

1. Prepare the kinase buffer: $50 \mathrm{mM}$ HEPES-NaOH pH 7.5 (HEPES stands for 4-(2-hydroxyethyl)-1-piperazineethanesulfonic acid), $150 \mathrm{mM} \mathrm{NaCl}, 5 \mathrm{mM} \mathrm{MgCl}_{2}$, $5 \mathrm{mM} \mathrm{MnCl}_{2}, 50 \mathrm{mM} \mathrm{NaF}, 1 \mathrm{mM} \mathrm{Na} \mathrm{VO}_{4}, 20 \mathrm{mM}$ $\beta$-glycerophosphate, $1 \mu \mathrm{g} / \mathrm{mL}$ leupeptin, and $1 \mathrm{mM}$ PMSF. Around $2 \mathrm{~mL}$ of the kinase buffer is required for each immunoprecipitation condition.

2. Wash immunoprecipitated beads from step 4.7 twice with $500 \mu \mathrm{L}$ of lysis buffer.

1. Centrifuge for $2 \mathrm{~min}$ at $1,000 \mathrm{xg}$ in a refrigerated centrifuge at $4^{\circ} \mathrm{C}$.

2. Carefully remove supernatant, and discard it. Add $500 \mu \mathrm{L}$ of lysis buffer. Homogenize by inversion.

3. Repeat steps 6.2.1 and 6.2.2.

3. Wash immunoprecipitated beads three times with $500 \mu \mathrm{L}$ of kinase buffer.

1. Centrifuge for $2 \mathrm{~min}$ at $1,000 \mathrm{x} \mathrm{g}$ in a refrigerated centrifuge at $4^{\circ} \mathrm{C}$.
2. Carefully remove supernatant, and discard it. Add $500 \mu \mathrm{L}$ of kinase buffer. Homogenize by inversion

3. Repeat steps 6.3.1 and 6.3.2 twice.

4. Centrifuge for $2 \mathrm{~min}$ at $1,000 \mathrm{xg}$ in a refrigerated centrifuge at $4{ }^{\circ} \mathrm{C}$.

5. Carefully remove supernatant, and discard it. Remove the last drops of supernatant with a Hamilton syringe in order to avoid aspiration of the beads, and discard the supernatant.

6. Add $40 \mu \mathrm{L}$ of kinase buffer to the immunoprecipitated beads. Resuspend the beads by gently tapping the tube.

7. Prepare the mix for the $y\left[{ }^{32} \mathrm{P}\right]$ ATP labeling (final volume is $22.5 \mu \mathrm{L}$, complete with kinase buffer) in a safe lock-tube.

1. Add the required volume of kinase buffer to reach a final volume of $22.5 \mu \mathrm{L}$.

2. Cut the end of a $20 \mu \mathrm{L}$ tip of a micropipette. Resuspend the immunoprecipitated beads from step 6.6 by pipetting up and down several times. Collect $10 \mu \mathrm{L}$ of these beads into the safe-lock tube.

3. Add ATP from a $10 \mu \mathrm{M}$ stock solution to reach $50 \mathrm{mM}$ of finale concentration. According to the number of sample treated, a dilution of the stock solution of ATP in the kinase buffer is suggested to allow to pipette 1 to $2 \mu \mathrm{L}$, to be sure the volume is correct.

4. Add $2.5 \mu \mathrm{g}$ of substrate (cofilin or Myelin Basic Protein, MBP in the present study case).

8. Add $5 \mu \mathrm{Ci}$ of $\gamma\left[{ }^{32} \mathrm{P}\right] \operatorname{ATP}(3,000 \mathrm{Ci} / \mathrm{mmol})$ to initiate the reaction. Mix by pipetting up and down slowly. 
CAUTION: From this point, work must be done in a safety place dedicated for radioactivity manipulations with cautious precaution, dedicated protections and appropriate controls (radioactive shields, Geiger counter, specific waste collect, personal breast and finger badges to detect radioactive exposure, filter tips).

9. Incubate for $20 \mathrm{~min}$ at $30^{\circ} \mathrm{C}$.

10. Stop the reaction with $6 \mu \mathrm{L}$ of $5 \mathrm{x}$ Laemmli buffer.

11. Heat at $95^{\circ} \mathrm{C}$ for $5 \mathrm{~min}$.

12. Centrifuge at $10,000 \mathrm{xg}$ for $5 \mathrm{~min}$ at room temperature.

13. Load on a SDS-PAGE. Proceed to migration.

NOTE : Take care that the front line of free $\mathrm{y}\left[{ }^{32} \mathrm{P}\right]$ ATP does not exit the gel to avoid contamination of the migration tank.

14. Stain the gel at room temperature.

1. Remove the gel from the glass plates.

2. Proceed with three baths in water at room temperature.

3. Stain the gel overnight with Coomassie Blue at room temperature.

4. Destain the gel with several wash baths with water at room temperature.

15. Wrap the gel with plastic wrap.

16. Expose for one night or more on a phosphorimager screen.

17. Read the screen on a phosphorimager to detect labeled bands.

\section{Representative Results}

\section{LIMK2-1 protein is synthesized}

LIMK2-1 is mentioned in databanks, but thus far only one paper has shown the existence of its mRNA ${ }^{18}$. Compared to its two homologs, LIMK2a and LIMK2b, LIMK2-1 has an extra C-terminal domain identified as a Protein Phosphatase 1 Inhibitory domain (PP1i). We designed an antibody that targets a peptide of this domain, amino acids 671-684 (Figure 1A).

BLAST research against human protein databases showed that only one protein, PHI-1 (Phosphatase holoenzyme inhibitor 1), has a strong sequence similarity with this 12 amino acid sequence. However, PHI-1 migrates at $23 \mathrm{kDa}$ on SDS-PAGE gels, far away from LIMK2-1, which is expected to migrate around $75 \mathrm{kDa}$ (i.e., the two should not interfere). We validated this antibody firstly for HEK-293 cells transfected either with LIMK2-1, LIMK2a, or LIMK2b and showed that the anti-PP1i antibody was able to recognize transfected LIMK2-1 (pCMV-LIMK2-1) and HA-tagged LIMK2-1, but did not cross-react with transfected HA-tagged LIMK2a or LIMK2b (Figure 1B). We observed a band of endogenous LIMK2-1 in HEK cells transfected with HA-tagged versions of the LIMK2 isoforms (indicated by an arrow in the anti-PP1i blot; Figure 1B). Secondly, we checked whether the signal induced by the anti-PP1i antibody was specific to LIMK2-1 using siRNA targeting the three spliced variants of LIMK2 (Figure 1C). In the presence of LIMK2 siRNA, a reproducible and significant decrease in the band of interest was observed (indicated by an arrow in Figure 1C) compared to control conditions, suggesting that the antibody is specific to LIMK2-1. Following this, we used the anti-PP1i antibody to detect LIMK2-1 in different cell line extracts: HEK-293 (Human Embryonic Kidney cells), HeLa (Human Epithelial Cervix cells), and C6 (Rat Brain Glial cells). These cells are disrupted in the lysis buffer containing 1\% Triton X-100. Using in silico analysis, LIMK2-1 
was shown to be Hominidae primate-specific ${ }^{19}$. Western Blot analysis using the anti-PP1i antibody showed that LIMK2-1 appeared to be expressed in HEK-293 and HeLa but not in C6 cell lines as expected from in silico studies (Figure 1D). These experiments were repeated with various human tissues, showing that levels of LIMK2-1 protein varied depending on the tissue: the highest levels were found in liver, lesser levels in pancreas, and the lowest in the testis and lung. LIMK2-1 was not detectable in brain tissue (Figure 1E). We observed lower molecular weight bands in all tissue samples except liver, suggesting the degradation of the full protein probably due to the lysis conditions of these commercial samples (this lysis buffer contains a cocktail of inhibitors not specified on the data sheet, which may be less efficient than the numerous protease and phosphatase inhibitors we are using in our home-made buffer). These data show that human LIMK2-1 protein is synthesized and expressed differently in the tested tissues.

\section{LIMK2-1 interacts with its upstream kinase ROCK}

LIMK2-1 homologs, LIMK2a and LIMK2b, have been described as regulated by the upstream kinase ROCK. We assessed the interaction of LIMK2-1 with ROCK using coimmunoprecipitation experiments. HEK cells were co-transfected with vectors encoding cMyc-tagged ROCK1 and either one of the HA-tagged version of LIMK2 isoforms, or the unrelated HA-tagged protein Larp6. Larp6 serves as a negative control, permitting the detection of non-specific interactions. Cells were lysed and anti-HA immunoprecipitation was performed. Lysates (whole cell extracts) and immunoprecipitates were analyzed by Western Blot, using anti-HA and anti-cMyc antibodies. As depicted in Figure 2 (left panels; Lysates), each of the different proteins encoded by the transfected vectors are well expressed. The three isoforms of LIMK2, as well as Larp6, are efficiently immunoprecipitated (Figure 2, bottom right panel; Eluates). In the eluates, ROCK is detected and thus coimmunoprecipitated with the three isoforms of LIMK2, but not with Larp6 (Figure 2, top right panel; Eluates). This demonstrates that ROCK interacts with the three isoforms of LIMK2, especially with the newly characterized isoform LIMK2-1. This interaction is specific since the negative control (Larp6) does not interact with ROCK.

Herein, we present data for the immunoprecipitation performed with anti-HA antibodies; however, the interaction may be tested in the opposite direction by immunoprecipitating ROCK with beads conjugated with $\mathrm{cMyc}$ antibodies and analyzing eluates with HA antibodies to detect LIMK coimmunoprecipitation.

\section{Kinase activity}

Phospho-cofilin in intact cells

The homologs of LIMK2-1, LIMK2a and LIMK2b, have been shown to phosphorylate cofilin, an actin depolymerisation factor, on its Serine3. Using an antibody specifically targeting the phospho-Serine3 of cofilin, we studied LIMK2 kinase activity by measuring the level of endogenous phospho-cofilin in HEK cells overexpressing one of the LIMK2 isoforms. HEK cells were transfected with vectors encoding either one of the HA-tagged LIMK2 isoforms, or the corresponding empty vector as a negative control. Cells were lysed, and the different lysates were analyzed by Western Blotting using the anti-phospho-Serine3 cofilin antibody. The overexpression of LIMK2a and LIMK2b induced a significant and reproducible increase in phospho-cofilin levels relative to control conditions, whereas the presence of LIMK2-1 had no detectable effect (Figure 3, left panel).

We repeated the same experiment with a C-terminal YFP-tagged (Yellow Fluorescent Protein) version of the three LIMK2 isoforms and an untagged version of LIMK2-1 to rule out possible interference by the N-terminal HA tag. The results were identical to those obtained using HAtagged versions of 
the three isoforms (Figure 3, right panel showing the YFP tagged version). Transfection efficiency was assessed for the YFPtagged version of LIMK isoforms using flow cytometry to rule out that this result may have been due to difference of protein expression. The three isoforms showed similar transfection efficiency: 54\% for LIMK2-1, 49\% for LIMK2b and 43\% for LIMK2b.

In vitro kinase tests

We then studied the kinase activity of the LIMK2 isoforms by in vitro labeling with $\gamma\left[{ }^{32} \mathrm{P}\right]$ ATP. Figure 4A shows the general scheme of this in vitro labeling. HEK cells were transfected with either one of the HA-tagged versions of the LIMK2 isoforms or the unrelated protein, Larp6, which was used as a negative control. The kinase activity of the anti-HA immunoprecipitates was measured using recombinant GST-cofilin as a substrate in the presence of $\gamma\left[{ }^{32} \mathrm{P}\right]$ ATP. HA-immunoprecipitated Larp6 showed no kinase activity on cofilin. HA-immunoprecipitated LIMK2a and LIMK2b phosphorylated cofilin, whereas LIMK2-1 did not (Figure 4B). We obtained similar results using YFP-tagged versions of the three isoforms

immunoprecipitated with GFP-trap beads in the presence of recombinant cofilin and $\gamma\left[{ }^{32} \mathrm{P}\right]$ ATP (Figure 4D).

We then tested whether LIMK2-1 had no kinase activity or if its activity on cofilin was impaired. We repeated the in vitro labeling experiment using Myelin Basic Protein (MBP), an efficient substrate for numerous protein kinases, instead of cofilin. There was a high background signal in the control condition when the assay was performed in the presence of HA-tagged versions: the negative control, HA-immunoprecipitated Larp6, produced a strong signal of phosphorylated MBP, although it is not a kinase (Figure $\mathbf{4 C}$ ). We overcame this problem using the YFP-tagged version of these proteins. Under these conditions, the background in the control (YFP alone) was low, allowing more specific studies to be conducted.
GFP-trapped YFP-LIMK2a, LIMK2b, and LIMK2-1 showed kinase activity towards MBP, although the activity of LIMK2-1 was lower (Figure 4D). However, LIMK2-1 was also less efficiently immunoprecipitated under these conditions (see Coomassie Brilliant Blue (CBB) staining and Western Blotting). Thus, the three isoforms showed comparable activity on MBP when phospho-MBP was normalized to immunoprecipitated LIMK2 levels by CBB staining (Figure 4D, lower panel). Immunoprecipitates were also analyzed by Western Blot using anti-ROCK antibodies to check if the activity on MBP could be due to the presence of ROCK, which would have been coimmunoprecipitated with LIMK2s. We could not detect any ROCK signal in LIMK2 immunoprecipitates. So MBP phosphorylation is not due to ROCK but to LIMK2s per se.

Overall, these data show that LIMK2a and LIMK2b have similar activities on cofilin and MBP. Although LIMK2-1 shows kinase activity towards MBP comparable to that of the other two isoforms, cofilin is not a good substrate for it.

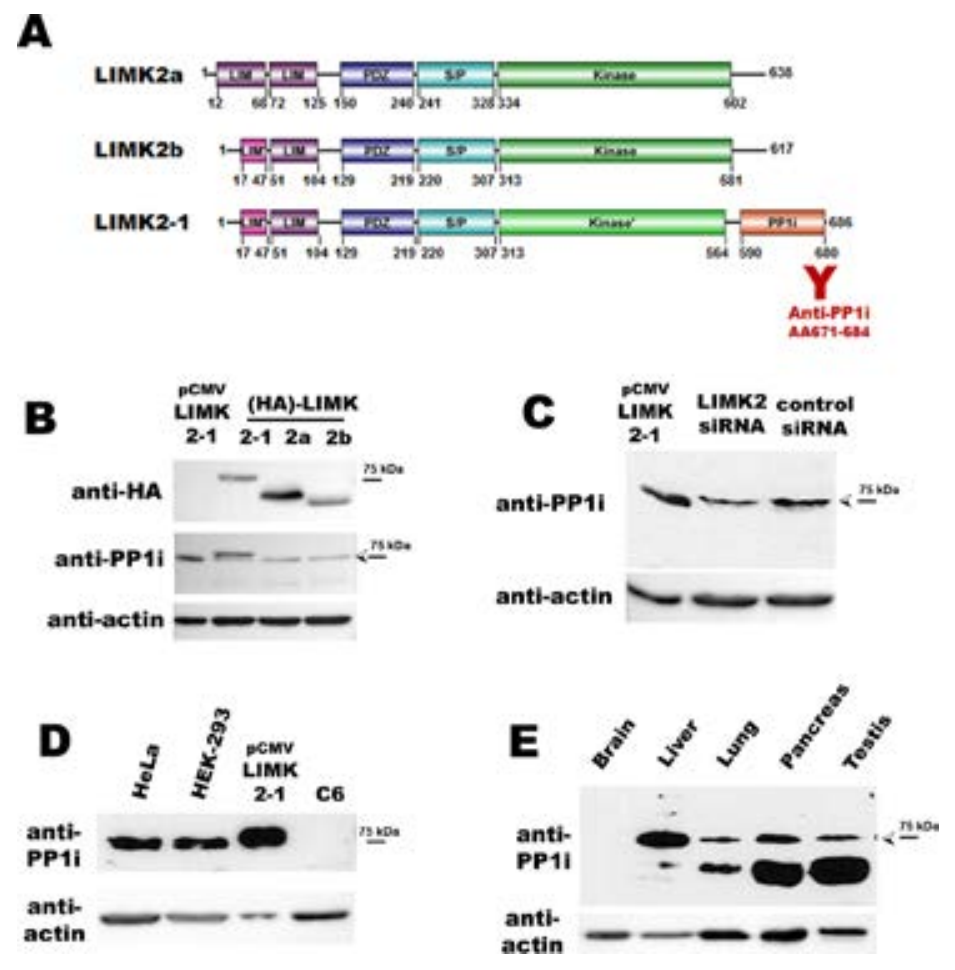


Figure 1: Evidence for the existence of the LIMK2-1 protein. (A) Schematic diagram of the three isoforms of human LIMK2. LIMK2 isoforms are described in Entrez Gene: LIMK2-1 (NP_001026971.1), LIMK2a (NP_005560.1), LIMK2b (NP_057952.1). The various domains of LIMK2 are shown: LIM (Lin11, IsI1, Mec3), LIM' (shorter LIM domain), PDZ (PSD95, Dlg1, Zo-1), S/P (Serine Proline rich), Kinase' (shorter kinase domain), and PP1i (Protein Phosphatase 1 inhibitory). The sequence chosen for anti-PP1i antibody design is shown in red. (B and C) Validation of the anti-LIMK2-1 antibody. HEK-293 cells were transfected with untagged LIMK2-1 (pCMV-LIMK2-1) or one of the HA-tagged isoforms of LIMK2. Lysates were analyzed by Western Blotting using the indicated antibodies. (C) HEK-293 cells were transfected either with LIMK2 siRNA or control siRNA. Lysates were analyzed by Western Blot. LIMK2-1 is expressed in various human cell lines (D) and tissues (E). HEK-293, HeLa and C6 cells were disrupted in 1\% Triton-X100 lysis buffer. Tissue extracts were purchased and samples thereof were analyzed by Western Blotting. This figure was modified from Vallee et al., The Biochemical Journal, 2018, http://www.biochemj.org/content/475/23/3745.long20.

click here to view a larger version of this figure.

ROCK1 +

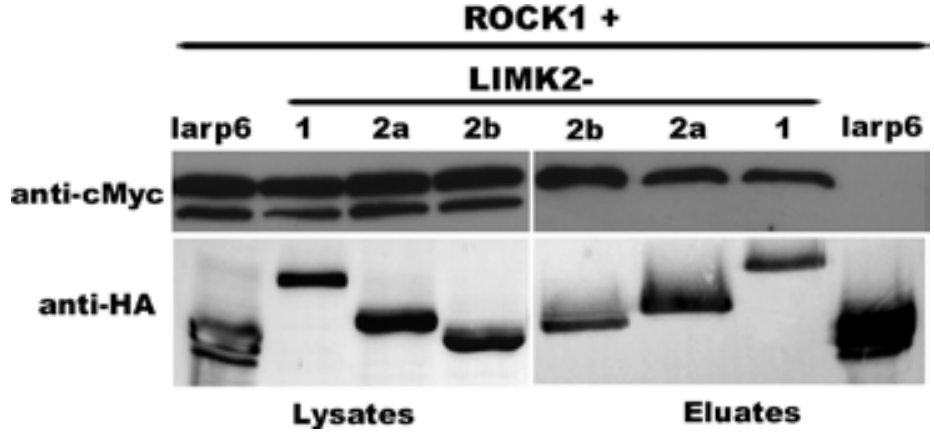

Figure 2: The three isoforms of LIMK2 interact with ROCK1. HEK-293 cells were co-transfected with cMyc-tagged ROCK1 and either one of the three HA-tagged LIMK2 isoforms (2-1, 2a, 2b) or the unrelated protein, Larp6. Lysates and anti-HA immunoprecipitates were subjected to Western Blotting.

click here to view a larger version of this figure.

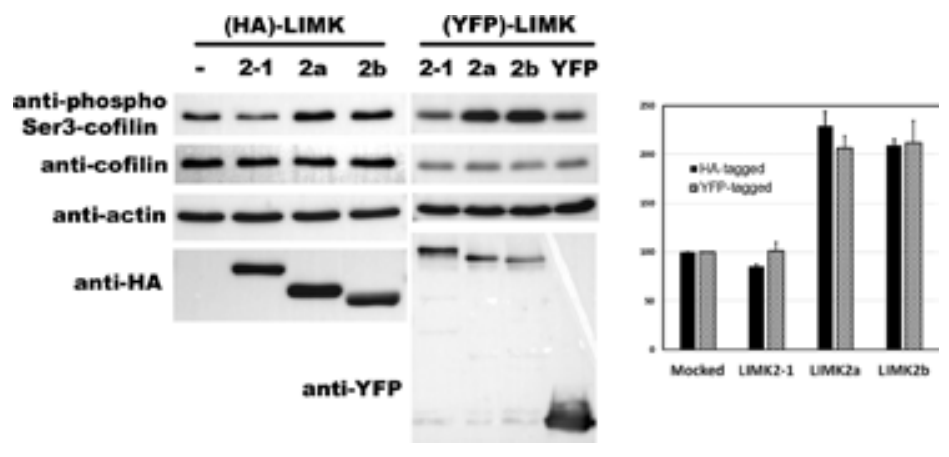

Figure 3: LIMK2-1 has no kinase activity towards cofilin in intact cells. HEK-293 cells were transfected with either one of the three HAtagged LIMK2 isoforms $(1,2 a, 2 b)$ or the empty parental vector, pcDNA3 (left panel), or with either one of the three YFP-tagged LIMK2 isoforms or YFP alone (right panel). Lysates were subjected to Western Blotting. Quantification of the ratio of phospho-cofilin versus cofilin is shown in the graph on the right. The phospho-cofilin versus cofilin ratio of mock transfected cells was normalized to 100 . Each value represents the mean \pm SE of three independent experiments. This figure was modified from Vallee et al., The Biochemical Journal 2018, http://www.biochemj.org/content/475/23/3745.long20. click here to view a larger version of this figure. 


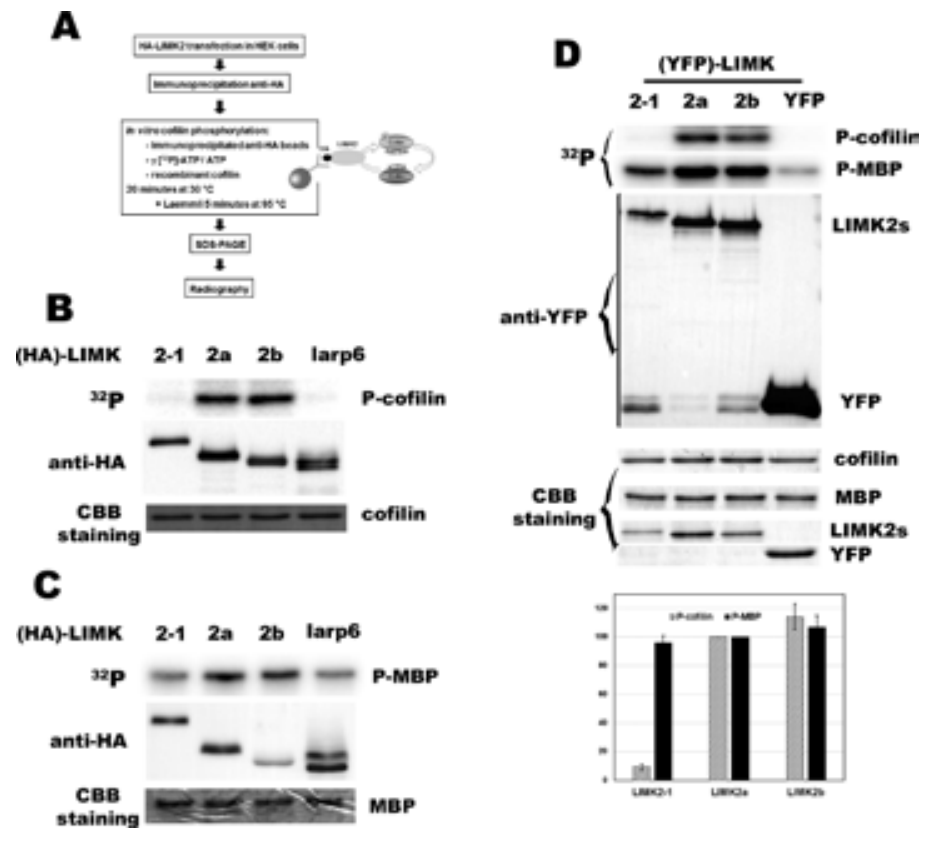

Figure 4: LIMK2-1 has no in vitro kinase activity towards cofilin, although it phosphorylates MBP. (A) General scheme of $\gamma\left[{ }^{32} \mathrm{P}\right]$ ATP in vitro labeling. (B) LIMK2-1 does not phosphorylate cofilin in vitro. HEK-293 cells were transfected with either one of the three HA-tagged LIMK2 isoforms (2-1, 2a, 2b) or an unrelated HA-tagged protein, Larp6, as a negative control. Anti-HA immunoprecipitated proteins and GSTcofilin were used in the kinase assay. The anti-HA immunoprecipitates were also subjected to anti-HA immunoblotting and Coomassie blue staining. (C) HA-tagged immunoprecipitation has a strong background signal when MBP is used as a substrate. HEK-293 cells were transfected with either one of the three HA-tagged LIMK2 isoforms (2-1, 2a, 2b) or an unrelated HA-tagged protein, Larp6, as a negative control. Anti HA immunoprecipitated proteins and MBP were used in the kinase assay. The anti-HA immunoprecipitates were also subjected to anti-HA immunoblotting and Coomassie blue staining. (D) The three LIMK2 isoforms have kinase activity towards Myelin Basic Protein (MBP). HEK-293 cells were transfected with either one of the three YFP-tagged LIMK2 isoforms $(2-1,2 \mathrm{a}, 2 \mathrm{~b})$ or YFP alone.
Anti-GFP immunoprecipitated LIMK2 isoforms and cofilin or MBP were used in the kinase assay. The anti-GFP immunoprecipitates were also subjected to anti-GFP immunoblotting and Coomassie blue staining. Quantification of phospho-cofilin and phospho-MBP is shown in the bottom graph. Phospho-cofilin levels obtained with anti-GFP immunoprecipitated LIMK2a were normalized to 100. Each value represents the mean \pm SE of three independent experiments. This figure was modified from Vallee et al., The Biochemical Journal, $2018, v$

http://www.biochemj.org/content/475/23/3745.long20.

click here to view a larger version of this figure.

\section{Discussion}

Herein, we have used robust biochemical tools to characterize at the molecular level a new protein, LIMK2-1, believed to be a kinase based on its sequence and on its homologs, LIMK2a and LIMK $2 b^{20}$.

Firstly, we demonstrated the existence of LIMK2-1 at the protein level using Western Blot analysis with a specific antibody. Following this, we evaluated its interaction with the upstream kinase ROCK1, which is known to regulate LIMK2a and LIMK2b, the homologs of LIMK2-1. Finally, we assessed the potential kinase activity of LIMK2-1 through in vitro $\gamma\left[{ }^{32} \mathrm{P}\right]$ ATP labeling and in cellulo by Western Blot using a specific phosphoantibody.

Lysis buffer composition

When studying proteins in order to analyze them by Western Blot, particular care is required with respect to lysis buffer composition. Several parameters have to be considered: (i) detergent type and concentration ${ }^{21}$, and (ii) protease inhibitors. 
The lysis buffer composition must be adapted to the target protein to facilitate its near complete solubilization in order to extract it as much as possible and to allow its detection by Western Blot. For soluble proteins, mild conditions (e.g., a mild detergent at low concentration) are often sufficient to achieve this. For membrane proteins, stronger conditions are typically often required. Different categories of detergents exist: (i) ionic, such as sodium dodecyl sulfate (SDS), cetyltrimethylammonium bromide (CTAB), (ii) non-ionic such as Polyethylene glycol hexadecyl ether (BRIJ), Triton, octylGlucoside (OG), doDecylMaltoside (DDM), and (iii) zwitterionic such as 3-[(3-Cholamidopropyl)dimethylammonio]-1propanesulfonate (CHAPS) or zwittergents. Strong detergents may disrupt interactions and complexes may be lost. Moreover, for immunoprecipitation experiments, antibodies may be sensitive to severe conditions. Similarly, enzyme activity may be disturbed by the presence of detergents that may unfold or denature the studied protein. Both the type and quantity of the detergent used may affect the properties and the activity of a protein. For some proteins, a very restricted range of concentration of detergent is tolerated to preserve the activity of the protein. Below this range the protein remains insoluble whereas above this range of concentration, the protein is no longer active.

Protease inhibitors must be added to the lysis buffer to prevent degradation of the target protein by endogenous proteases. Protease inhibitor cocktails are commercially available. They can be used as a starting point of a study. If troubles are encountered, one may consider the use of a mixture of inhibitors extemporarily prepared from stock solutions stored at $-20{ }^{\circ} \mathrm{C}$. These inhibitors must target serine and cysteine proteases. PMSF (Phenylmethylsulfonyl fluoride) is commonly used, however it is very unstable in aqueous solution and must be added just before the extraction. Metalloproteases should also be inhibited: metal chelating reagents, such as EDTA (Ethylenedinitrilotetraacetic acid) or EGTA (Ethylene glycol-bis(2-aminoethylether)-tetraacetic acid), which bind to $\mathrm{Mg}^{2+}$, are used in this purpose. To keep protein in their phosphorylated and thus activated form, it is also recommended to add phosphatase inhibitors to the lysis buffer. These inhibitors must target alkaline, acid, serine, threonine, and tyrosine phosphatases. Working at low temperature $\left(4{ }^{\circ} \mathrm{C}\right)$ is also recommended in order to slow down the rate of proteolysis.

\section{Target proteins}

Herein, we focused on epitope-tagged proteins. Tags (Flag, HA, cMyc, GFP, etc.) are very useful in order to detect and purify proteins, as antibodies and antibody coupled beads are commercially available and are readily reproducible materials. However, the size and the position of the tag has to be considered as this may affect the activity, localization or function of the target protein $6,7,8$. It is also possible to work with endogenous proteins. In this case, antibodies targeting this particular protein must be used. They may be coupled to beads (Protein A or Protein G) covalently (cross-link) or be incubated with the lysate and then with the beads. When working with tagged proteins, whose gene is expressed on a plasmid, it is easy to switch to a mutated version of this protein by mutagenesis of the gene. It is then possible to work on different mutants to assess biological functions.

\section{Immunoprecipitation}

Immunoprecipitation is a very powerful technique to isolate partners of the target protein ${ }^{5}$. The composition of the lysis buffer (especially detergent) has to be carefully established to preserve interactions (see above). It is possible to detect the interaction between two identified proteins. It may be endogenous proteins or overexpressed proteins. When proteins are expressed at low abundance, it may be necessary to 
overexpress them in order to have stronger signals. Extensive washes of immunoprecipitated beads are required to remove non-specifically interacting proteins or contaminants. Prior to determining immunoprecipitation efficiency or co-immunoprecipitated partner presence, it is important to check that the different partners are well expressed and present in the lysate by analyzing the whole cell lysate or the input fraction by Western Blot. Furthermore, using immunoprecipitation experiments, it is also possible to identify new partners of a target protein and to isolate new complexes. These new partners may be identified by mass spectrometry.

Such partners may play an important role as activators of the target protein allowing its full activity for further biological tests. On the other hand, copurified unwanted proteins may be used as an argument that there is not a direct interaction between two identified partners, but instead that the detected interaction by co-immunoprecipitation is due to another unknown partner. In this specific case, to be sure of a direct interaction, another experimental device is needed, such as working on mammalian proteins purified from bacteria.

\section{Kinase activity}

Kinase activity may be assessed by different techniques. Herein, we focused on in vitro analysis by $\gamma\left[{ }^{32} \mathrm{P}\right]$ ATP labeling and on whole cell extract analysis by Western Blot using a specific phospho-antibody. $\gamma\left[{ }^{32} \mathrm{P}\right]$ ATP labeling is a very sensitive and quantitative technique allowing the detection of weak kinase activity ${ }^{15}$. Radioactivity incorporation from ATP to the target substrate allows a direct measure of enzyme activity to be made. It is possible to work with different substrates to assess the kinase activity of the studied protein on different targets. It is also possible to identify amino acids crucial for kinase activity by mutating them when the protein is overexpressed. Kinase activity requires a divalent cation such as $\mathrm{Mg}^{2+}$, which has to be present in the kinase buffer.
The major drawback of this approach is the handling of radioactivity, which requires dedicated facilities for experiments and for the collection of wastes. Alternative methods exist, such as fluorescent or luminescent kits that detect byproducts of the reaction, such as ADP (adenosine diphosphate) ${ }^{16}$. Protein phosphorylation may also be studied by mass spectrometry, however, a larger amount of materials is required for these analyses. In our case, we tried to assess LIMK2 kinase activity using capillary electrophoresis to increase our efficiency but this was unfortunately unsuccessful.

Phosphospecific antibodies are a further tool to study the phosphorylation of a protein. Broad general anti-phospho Serine and Tyrosine antibodies exist, able to recognize phospho-Ser or phospho-Tyr of any proteins. In recent years, antibodies targeting a specific phospho-site of a target protein have been widely developed and usually they recognize both the phospho group and the surrounding amino acids. Special care is needed when starting to work with such antibodies, as their specificity must be checked for example with a negative control, such as the target protein mutated on the phospho site. When probing a blot with an anti-phospho antibody, the blocking solution must not be milk, as this contains phosphoproteins that may interact with the antibody. Bovine Serum Albumin (BSA) is recommended, and phosphatase inhibitors may be added in the blocking solution to prevent phosphate release. Samples should not be frozen and thawed, but rather prepared as aliquots which are kept at $-80^{\circ} \mathrm{C}$. Indeed, phospho modifications are labile.

\section{Disclosures}

The authors have nothing to disclose. 


\section{Acknowledgments}

This work was supported by La Ligue contre le Cancer, l'Association Neurofibromatoses et Recklinghausen, and la Région Centre Val de Loire. Many thanks to Aurélie Cosson and Déborah Casas for flow cytometry data, and to Keyron Hickman-Lewis for thorough proofreading of the manuscript.

\section{References}

1. Manning, G., Plowman, G. D., Hunter, T., Sudarsanam, S. Evolution of protein kinase signaling from yeast to man. Trends in Biochemical Sciences. 27, 514-520 (2002).

2. Manning, G., Whyte, D. B., Martinez, R., Hunter, T., Sudarsanam, S. The protein kinase complement of the human genome. Science (New York, N.Y.). 298, 1912-1934 (2002).

3. Ochoa, D., Bradley, D., Beltrao, P. Evolution, dynamics and dysregulation of kinase signalling. Current Opinion in Structural Biology. 48, 133-140 (2018).

4. Bhullar, K. S. et al. Kinase-targeted cancer therapies: progress, challenges and future directions. Molecular Cancer. 17, 48 (2018).

5. Kaboord, B., Perr, M. Isolation of proteins and protein complexes by immunoprecipitation. Methods in Molecular Biology (Clifton, N.J.). 424, 349-364 (2008).

6. Arnau, J., Lauritzen, C., Petersen, G. E., Pedersen, J. Current strategies for the use of affinity tags and tag removal for the purification of recombinant proteins. Protein Expression and Purification. 48, 1-13 (2006).

7. Wood, D. W. New trends and affinity tag designs for recombinant protein purification. Current Opinion in Structural Biology. 26, 54-61 (2014).
8. Young, C. L., Britton, Z. T., Robinson, A. S. Recombinant protein expression and purification: a comprehensive review of affinity tags and microbial applications. Biotechnology Journal. 7, 620-634 (2012).

9. Greenwood, C. et al. Proximity assays for sensitive quantification of proteins. Biomolecular Detection and Quantification. 4, 10-16 (2015).

10. Wang, X., Huang, L. Dissecting Dynamic and Heterogeneous Proteasome Complexes Using In vivo Cross-Linking-Assisted Affinity Purification and Mass Spectrometry. Methods in Molecular Biology (Clifton, N.J.). 1844, 401-410 (2018).

11. Raykova, D. et al. Let There Be Light! Proteomes. 4 (2016).

12. Weibrecht, I. et al. Proximity ligation assays: a recent addition to the proteomics toolbox. Expert Review of Proteomics. 7, 401-409 (2010).

13. Podobnik, M. et al. How to Study Protein-protein Interactions. Acta Chimica Slovenica. 63, 424-439 (2016).

14. Rao, V. S., Srinivas, K., Sujini, G. N., Kumar, G. N. Protein-protein interaction detection: methods and analysis. International Journal of Proteomics. 2014, 147648 (2014).

15. Peck, S. C. Analysis of protein phosphorylation: methods and strategies for studying kinases and substrates. The Plant Journal: For Cell and Molecular Biology. 45, 512-522 (2006).

16. Jia, Y., Quinn, C. M., Kwak, S., Talanian, R. V. Current in vitro kinase assay technologies: the quest for a universal format. Current Drug Discovery Technologies. 5, 59-69 (2008). 
17. Okano, I. et al. Identification and characterization of a novel family of serine/threonine kinases containing two N-terminal LIM motifs. The Journal of Biological Chemistry. 270, 31321-31330 (1995).

18. Croft, D. R. et al. p53-mediated transcriptional regulation and activation of the actin cytoskeleton. Cell Research. 21, 666-682 (2011).

19. Tastet, J. et al. LIMK2-1 is a Hominidae-Specific Isoform of LIMK2 Expressed in Central Nervous System and Associated with Intellectual Disability. Neuroscience. 399, 199-210 (2019).

20. Vallee, B. et al. LIMK2-1, a new isoform of human LIMK2, regulates actin cytoskeleton remodeling via a different signaling pathway than that of its two homologs, LIMK2a and LIMK2b. The Biochemical Journal. 475, 3745-3761 (2018).

21. Lee, Y. C. et al. Impact of Detergents on Membrane Protein Complex Isolation. Journal of Proteome Research. 17, 348-358 (2018). 